\title{
La autoeficacia percibida del docente de Lenguas Extranjeras, desde un dispositivo estratégico y operativo de identificación de su profesionalización y desarrollo profesional
}

\author{
Francisco JaVier Sanz Trigueros \\ Carmen Guillén Díaz \\ Universidad de Valladolid
}

Recibido: 24 marzo 2015 / Aceptado: 23 julio 2015

ISSN: $1697-7467$

\begin{abstract}
RESUMEN: En este artículo presentamos un estudio inscrito en la problemática de la profesionalización y del desarrollo profesional de los docentes de Lenguas Extranjeras como objetos de evaluación para la acreditación, la certificación y la movilidad. Focalizamos la atención en el sentimiento de autoeficacia de esos docentes, a través de dos indicadores complementarios de la profesionalización: el grado de sensibilización ante su propio perfil profesional y las autopercepciones sobre las condiciones de configuración del desarrollo profesional. Optando por el paradigma cualitativo, los datos son obtenidos mediante la encuesta administrada a una muestra significativa invitada por situaciones docentes, y son analizados movilizando la técnica del análisis de contenido. Los resultados permiten aportar pautas orientadas a optimizar el desarrollo de las competencias profesionales docentes, en la perspectiva abierta por las determinaciones europeas al respecto.

Palabras Clave: Profesionalización docente, Perfil profesional del docente de Lenguas Extranjeras, Sentimiento de eficacia personal, Autoeficacia percibida, Análisis de contenido.
\end{abstract}

Foreign Languages' Teacher Self-Efficacy, from a Strategic and Operational Identification Device of Their Professionalization and Professional Development

\begin{abstract}
In this paper we present a research inscribed on the issue of professionalization and professional development of Foreign Languages' teachers which are shown as objects of assessment for accrediting, certification and mobility. We focus attention on the self-efficacy beliefs of these teachers through two complementary indicators: Their degree of awareness towards their own professional profile and their self-perceptions on the conditions shaping their professional development. Drawing on the qualitative paradigm, the data were obtained through a survey conducted amongst a significant invited sample composed of teaching situations, as well as analyzed by mobilizing the technique of content analysis. The findings permit providing guidelines optimizing the development of teacher professional competences.
\end{abstract}

Key Words: Teacher professionalization, Foreign Language Teacher professional Profile, Self-efficacy Beliefs, Perceived Self-efficacy, Content Analysis. 


\section{INTRODUCCIÓN}

Esta investigación exploratoria surge ante los desafíos y responsabilidades que, en nuestro contexto nacional generan dos órdenes esenciales para atender a la profesionalización de los docentes, en particular de Lenguas Extranjeras (en adelante LE): a) el de las determinaciones europeas al respecto y b) el de los componentes y caracterización de su desarrollo profesional.

Por lo que se refiere al orden a) de las determinaciones europeas, partimos de orientaciones comunes en materia de profesionalización docente proporcionadas por referentes institucionales que caracterizan, a su vez, la entidad e identidad profesional de estos docentes. Referentes que:

- por una parte, pretenden dar respuesta a uno de los retos pendientes para Europa en el horizonte del 2020, el de "Mejorar la calidad y la eficacia de la educación y la formación" (segundo objetivo estratégico, C119/3), concretado en lograr la profesionalización y el desarrollo profesional docente; y

- por otra parte, emanan de las políticas lingüísticas educativas europeas, nacionales, regionales y locales -según una coherencia vertical-, pues hay que reconocer que todo lo que concierne a la enseñanza de lenguas en los sistemas educativos ${ }^{1}$ ha de inscribirse, subsidiariamente, en las dinámicas europeas (Pilhion, 2008).

De forma que, por el papel primordial que desempeñan los docentes (Comisión Europea, 2005), se trata de conceder la prioridad a:

- Sus posibilidades de formación permanente y de desarrollo profesional continuo.

- Un reconocimiento apropiado de sus competencias profesionales especificadas en referenciales de competencias genéricas (Comisión Europea, 2008; Paquay, 2010; Tardif, 2004), y específicas propias de un perfil europeo del docente de LE (Kelly et al., 2004).

- La validación, evaluación, certificación y registro de sus cualificaciones (INCUAL, 2007).

- La necesidad de sensibilizar a estos docentes sobre la importancia que tiene para ellos participar activamente en su propio desarrollo profesional y reflexionar sobre sus competencias.

Y, por lo que se refiere al orden b) de los componentes y caracterización del desarrollo profesional del docente de LE, consideramos su complejidad desde la lógica del aprendizaje permanente (Tejada, 2013), su carácter procesual para la mejora continuada de la calidad docente, y su vinculación con la entidad e identidad profesionales (Beckers, 2007; Donnay y Charlier, 2008) que, en el marco de las teorías sociocognitivas, nos remite al sistema de componentes personales externos e internos, configuradores de un perfil profesional propio e implicados en dicho desarrollo profesional.

\footnotetext{
${ }^{1}$ Para los niveles organizativos curriculares de la enseñanza de LE, supra, macro, meso a los que alude la Guía para la elaboración de las políticas lingüisticas en Europa, http://www.coe.int/t/dg4/linguistic/guide_niveau3 _FR.asp-,
} 
En ese sistema propio, el sentimiento de eficacia personal es:

- concretado en la autoeficacia percibida (Bandura, 2006);

- considerado el fundamento de las actividades humanas, y clave en la adquisición de competencias y en los logros; y

- abordado como un mecanismo autorregulador central de la actividad humana, al residir en la creencia que posee un individuo en su capacidad para realizar o no una tarea, alimentándose de fuentes que permiten construirlo o modificarlo. Se pone de manifiesto y se enriquece, a su vez, a través de dos factores complementarios tomados aquí como indicadores clave de su autoeficacia percibida: el grado de sensibilización ante el propio perfil profesional y las autopercepciones sobre las condiciones de configuración del desarrollo profesional.

En la perspectiva última de contribuir a optimizar el proceso de profesionalización del docente de LE y las condiciones que inciden en su desarrollo profesional competencial, el objetivo general de este estudio es aportar conocimiento sobre ese proceso a través de los dos indicadores citados, para estar en condiciones de proponer pautas estimadas pertinentes porque optimizarían el desarrollo de las competencias profesionales docentes.

\section{Aspectos metodológicos de la inVestigación}

\subsection{Posicionamiento metodológico}

Para el ámbito de las creencias que nos ocupa, esta investigación se inscribe en los paradigmas cualitativo interpretativo (Flick, 2004; Gurdián-Fernández, 2007; Stake, 2010) y del pensamiento del profesor (Marcelo, 1987) ${ }^{2}$, que nos determinan a adoptar un enfoque descriptivo e interpretativo. Al tratarse de informaciones manifiestas -relativas a las creencias de los sujetos participantes sobre su perfil profesional y a las autopercepciones de eficacia personal-, para su tratamiento como datos, la técnica de la encuesta se presta para su recogida, pues van a aparecer explícita y/o implícitamente en las respuestas de un cuestionario; instrumento que concebimos y elaboramos como dispositivo estratégico y operativo.

Para el tratamiento de los datos, se ha contemplado la técnica global del análisis de contenido (Bardin, 2002; Krippendorff, 1990), mediante un sistema categorial organizado en torno al procedimiento de "casillas", coherente con el método deductivo utilizado.

\subsection{Los contextos y los sujetos participantes}

A la búsqueda de la operatividad y eficacia en la obtención de informaciones que aportasen datos significativos:

- La recogida de datos se localiza en contextos institucionales tipo de educación y formación, en donde los docentes de LE desempeñan su actividad, así como en la Universidad, institución en la que se forman los futuros docentes de LE.

\footnotetext{
${ }^{2}$ Este autor se ha centrado específicamente en los vínculos entre la teoría y las prácticas docentes.
} 
- La muestra poblacional se caracteriza por ser intencional e invitada; ubicada en Valladolid, está compuesta por 25 participantes de tres sectores de docentes que abarcan en su conjunto diferentes niveles y centros, así como tiempo de docencia desempeñado.

- Los criterios priorizados para la delimitación de los contextos y la selección de los participantes -desde nuestra posición de investigadores- han sido los siguientes:

- El fácil acceso y proximidad a los centros de orden educativo y de orden formativo.

- Los vínculos personales y las relaciones profesionales afables.

- La fuerte implicación y plena disposición de cada docente para participar, informar y aportar todos los datos requeridos, por contacto profesional indirecto.

Desde estas determinaciones:

- A los contextos de orden educativo localizados en Centros de Enseñanza Obligatoria corresponden, del total de la muestra, 11 sujetos pertenecientes al Sector de los docentes educadores en LE; 10 docentes de Educación Primaria y 1 docente de Educación Secundaria.

- A los contextos de orden formativo localizados en la Escuela Oficial de Idiomas (E.O.I.) y Academias de idiomas corresponden, del total de la muestra, 10 sujetos pertenecientes al Sector de los docentes formadores de LE; 5 docentes de E.O.I. y 5 de Academias de idiomas.

- Para el contexto localizado en la Universidad de Valladolid (UVa) contamos, del total de la muestra, con 4 sujetos pertenecientes al Sector de los futuros docentes de $L E$, que se encuentran al término del periodo profesionalizador del Máster en Profesor de Educación Secundaria Obligatoria y Bachillerato, Formación Profesional y Enseñanzas de Idiomas. Todos ellos hacen explícita su fuerte orientación a ejercer la docencia de LE en contextos de diverso orden.

\subsection{Para la concepción y elaboración del instrumento de recogida de datos}

El cuestionario, concebido como un dispositivo estratégico y operativo, ha sido elaborado y formalizado conforme a las fases canónicas de: a) Referencialización; b) Definición de los indicadores y Transposición en categorías de análisis para la enunciación de los items; d) Validación.

Para la referencialización, accedimos a los documentos innovadores generados ante las directrices europeas en materia de profesionalización de los docentes de LE.

Es el caso del Profil (Kelly et al., 2004), y del European Profiling Grid-EPG- (EAQUALS, 2013) del que retenemos los bloques y descriptores relativos a la profesionalización del docente de LE, y específicamente vinculados tanto al desarrollo profesional en la propia institución como a la competencia interpersonal, concretada en: Acción tutorial y orientación, feedback, mediación, mentoría, etc.

Al mismo tiempo dispusimos de los propuestos en nuestro contexto nacional y regional, como es el caso de: 
1) El Modelo de competencias profesionales del profesorado (Junta de Castilla y León, JCyL, 2011a), cuyas denominaciones están precedidas de su codificación y seguidas del número de descriptores que las definen:

- A. Competencia cientifica cognitiva (1 a 3$)$

- B. Competencia intra e interpersonal (4-7)

- C. Competencia didáctica (8-14)

- D. Competencia organizativa y de gestión del Centro (15 a 17)

- E. Competencia en gestión de la convivencia (18-20)

- F. Competencia en trabajo en equipo (21 a 24)

- G. Competencia en innovación y mejora (25 a 28)

- H. Competencia comunicativa y lingüistica (29 a 32)

- I. Competencia digital (TIC) (33 a 36)

- J. Competencia social-relacional (37 a 40)

2) El Plan de formación del profesorado en Lenguas Extranjeras (Junta de Castilla y León, JCyL, 2011b) que, articulado en tres dimensiones, propone un itinerario formativo por ámbitos, y respecto a una o varias de las competencias profesionales descritas en el Modelo (JCyL, 2011a), según ilustramos en el Cuadro 1.

Cuadro 1. Correspondencia entre las dimensiones y ámbitos formativos y competencias atendidas

\begin{tabular}{|c|c|c|c|}
\hline $\begin{array}{l}\text { Dimensiones } \\
\text { del Plan }\end{array}$ & Ámbitos de formación & Competencias & Descriptores \\
\hline \multirow[b]{2}{*}{$\begin{array}{l}\text { Dimensión } \\
\text { lingüístico- } \\
\text { comunicativa }\end{array}$} & - Las propias destrezas lingüísticas en LE & $\mathrm{H}$ & 32 \\
\hline & $\begin{array}{l}\text { - Conocimientos teóricos relativos a la } \\
\text { LE (Lingǘstica, Cultura, Adquisición de } \\
\text { una L2, etc.) }\end{array}$ & A & $1,2,3$ \\
\hline \multirow{4}{*}{$\begin{array}{l}\text { Dimensión } \\
\text { didáctica }\end{array}$} & $\begin{array}{l}\text {-Actualización e innovación } \\
\text { metodológica / didáctica }\end{array}$ & $\mathrm{C}$ & 10 у 25 \\
\hline & - Metodología AICLE/CLIL/EMILE & G & 28 \\
\hline & \multirow{2}{*}{ - Currículo integrado } & $\mathrm{C}$ & 8 \\
\hline & & $\mathrm{F}$ & 22 \\
\hline \multirow{4}{*}{$\begin{array}{l}\text { Dimensión } \\
\text { de desarrollo } \\
\text { profesional }\end{array}$} & - Programas europeos & $\mathrm{F}$ & 22 \\
\hline & \multirow{2}{*}{$\begin{array}{l}\text { - TIC en el Aula de idiomas/Trabajo } \\
\text { colaborativo en entornos virtuales }\end{array}$} & I & 33 y 34 \\
\hline & & $\mathrm{F}$ & 22 \\
\hline & - Portfolio Europeo de Lenguas (PEL) & $\mathrm{C}$ & 13 у 28 \\
\hline
\end{tabular}

Dichos ámbitos de formación conllevan el tiempo en función del número de horas asistidas (mínimo 10, máximo 60) y modalidad (presencial, semipresencial, online y seminario/jornada). 
El cuestionario resultante se articula en tres partes diferenciadas cuyos ítems corresponden a los componentes del sistema propio -el perfil-, que concebimos en relación con los referentes indicados como componentes del desarrollo profesional del docente de LE:

- Componente identificador: perfil profesional (Kelly et al., 2004).

- Componente formativo: ámbito, tiempo y modalidad. (JCyL, 2011b).

- Componente competencial: competencias profesionales docentes (JCyL, 2011a).

- Componente de evaluación: autoevaluación y acreditación (EAQUALS, 2013).

- Componente de eficacia: sensibilización ante el propio perfil profesional y autopercepciones de eficacia personal (Bandura, 2003).

Buscamos su pertinencia y adecuación como sistema categorial de referencia, para la determinación de las Categorías de análisis y su definición en Subcategorías que entroncan con los ámbitos de formación del Plan (JCyL, 2011b) y con las diversas competencias del Modelo (JCyL, 2011a). Ilustramos en:

Figura 1. Red de relaciones entre ítems del cuestionario, componentes y categorías de análisis

\begin{tabular}{|c|c|c|}
\hline Cuestionario & Componentes & Categorías de análisis \\
\hline PARTE 1 & Componente identificador & Indicadores de: \\
\hline Items $1-7$ & & \\
\hline PARTE 2 & $\begin{array}{l}\text { Componente formativo } \\
\text { Componente competencial }\end{array}$ & $\begin{array}{l}\text { 1. La Formación continua } \\
\text { del docente de LE y }\end{array}$ \\
\hline Items $13-14$ & Componente de evaluación & $\begin{array}{l}\text { 2. La Evaluación del } \\
\text { propio perfil profesional }\end{array}$ \\
\hline $\begin{array}{l}\text { PARTE } 3 \\
\text { Item } 15\end{array}$ & Componente de eficacia & 3 . I A Antoef \\
\hline $\begin{array}{l}\text { (30 enunciados } \\
1 \text { formulación) }\end{array}$ & & percibida \\
\hline
\end{tabular}

- la Figura 1, la red de relaciones que sustentan la concepción del cuestionario y, en consecuencia, la interpretación de las informaciones generadas;

- el Cuadro 2, la descripción de las Categorías y Subcategorías de análisis establecidas acompañadas de su codificación. 
Cuadro 2. Categorías de análisis 1, 2 y 3, subcategorías correspondientes y codificación

\begin{tabular}{|c|c|c|}
\hline Categorías de análisis & Subcategorías & Codificación \\
\hline \multirow{7}{*}{$\begin{array}{l}\text { 1. La Formación continua } \\
\text { del docente de LE }\end{array}$} & Destrezas lingüísticas & $d l$ \\
\hline & Conocimientos teóricos LE & $c t$ \\
\hline & $\begin{array}{l}\text { Actualización e innovación } \\
\text { metodológica/didáctica }\end{array}$ & imd \\
\hline & Metodología AICLE/CLIL/EMILE & $m a$ \\
\hline & Currículo integrado & $c i$ \\
\hline & Programas europeos & pe \\
\hline & TIC en el aula de idioma/entornos virtuales & $t v$ \\
\hline \multirow{13}{*}{$\begin{array}{c}\text { El desarrollo de competencias } \\
\text { profesionales }\end{array}$} & Portfolio Europeo de Lenguas & pel \\
\hline & Competencia científica cognitiva & $c c c$ \\
\hline & Competencia intra e interpersonal & cii \\
\hline & Competencia didáctica & $c \bar{d}$ \\
\hline & $\begin{array}{l}\text { Competencia organizativa y de gestión del } \\
\text { Centro }\end{array}$ & $\operatorname{cog}$ \\
\hline & Competencia en gestión de la convivencia & $c g c$ \\
\hline & Competencia en trabajo en equipo & cte \\
\hline & Competencia en innovación y mejora & cim \\
\hline & Competencia comunicativa y lingüística & $c c l$ \\
\hline & Competencia digital (TIC) & ctic \\
\hline & Competencia social-relacional & $\operatorname{cs} r$ \\
\hline & Acciones pasadas y/o actuales & apa \\
\hline & Acciones futuras & af \\
\hline \multirow[b]{2}{*}{$\begin{array}{l}\text { 2. La Evaluación } \\
\text { del propio perfil profesional }\end{array}$} & Programas e instrumentos de evaluación & $p i$ \\
\hline & Pruebas oficiales & po \\
\hline \multirow{6}{*}{ 3. La Autoeficacia percibida } & \multicolumn{2}{|l|}{ CP } \\
\hline & Competencia intra e interpersonal & $C / I I$ \\
\hline & $\begin{array}{l}\text { Competencia organizativa y de gestión del } \\
\text { Centro }\end{array}$ & $C / O G$ \\
\hline & Competencia en trabajo en equipo & $C / T E$ \\
\hline & Competencia en innovación y mejora & $C / I M$ \\
\hline & Competencia social-relacional & $C / S R$ \\
\hline
\end{tabular}

\subsection{Recogida y procesamiento de los datos}

Aplicado el cuestionario a los sujetos participantes conforme a los criterios indicados, movilizamos las operaciones sistemáticas del análisis de contenido, utilizando las parrillas que facilitan el tratamiento semántico manual de los datos de los tres sectores de informantes.

En las parrillas por unidades de análisis -cada componente relativo al desarrollo profesional docente-, y en función de las categorías de análisis (Cuadro 2), damos cuenta por unidades de contexto -Partes 1, 2 y 3 del cuestionario-, de las unidades de registro, de su 
frecuencia de aparición / intensidad, y de los códigos utilizados en la categorización y registro. En el caso de la categoría de análisis La Formación continua del docente de LE, y de la unidad de análisis Componente formativo, se consigna además la modalidad y el tiempo.

\section{Del anÁlisis de los datos a la interpretación de los Resultados POR COMPONENTES DEL PERFIL PROFESIONAL}

\subsection{El componente identificador}

Para este componente, el análisis de los datos nos permite describir que:

- En el sector de informantes docentes educadores en LE, cuya profesión se inscribe en el contexto de orden educativo, de los 11 encuestados de edades entre 27 y 50 años, 8 son mujeres y 3 hombres, con experiencia profesional docente entre 3 y 30 años y 6 meses. De los 3 hombres, uno imparte clase en Educación Secundaria Obligatoria, mientras que las 8 mujeres y 2 hombres en Educación Primaria. Todos poseen nacionalidad española y lengua materna español. Sus últimos estudios finalizados son: Diplomatura (6), Licenciatura (3), Grado (1) Máster (1) y Grado de Doctor (0).

- En el sector de informantes docentes formadores de LE, cuyo ejercicio profesional se inscribe en el contexto de orden formativo, 5 de los encuestados ejercen su profesión en E.O.I., y 5 en Academias de idiomas. Se trata de 8 mujeres (una considera como lenguas maternas el español y el portugués) y 2 hombres (uno con nacionalidad cubana). Sus edades oscilan entre 22 y 59 años y su experiencia profesional docente entre 5 meses y 30 años y 4 meses. Sus últimos estudios finalizados son: Licenciatura (5), Grado (3), Máster (1) y Doctorado (1).

- En el sector informantes futuros docentes -al término del período formativo del Máster Profesor de Educación Secundaria Obligatoria y Bachillerato, Formación Profesional y Enseñanzas de Idiomas, 3 son hombres de nacionalidad española sin experiencia en la docencia de LE; y 1 mujer de nacionalidad uzbeka (con el ruso como lengua materna) y con 14 años de experiencia profesional docente en LE. Sus edades oscilan entre 26 y 39 años y sus últimos estudios finalizados son: Grado (2), Posgrado (2) y Grado de Doctor (0).

\subsection{El componente formativo}

Del análisis de este componente formativo y con atención al ámbito, modalidad y tiempo se pueden observar tanto diferencias como aspectos comunes, respecto a los ámbitos de formación continua en los que se hallan implicados cada uno de los sectores de informantes. Cabe señalar que:

- El sector de informantes docentes formadores de LE es el único que cubre todos los ámbitos de formación continua, de entre los que el relativo a los programas europeos (pe) destaca considerablemente en el caso del sector de los docentes educadores. 
- Los tres sectores de informantes seleccionan unánimemente el ámbito de actualización e innovación metodológica/didáctica (imd), que destaca ampliamente de entre todos los ámbitos de formación continua que han marcado.

- De manera coincidente, los ámbitos de metodología AICLE/CLIL/EMILE ( $m a$ ) y el del PEL (pel) son los menos trascendentes para el sector de los docentes educadores $\mathrm{y}$ el de los docentes formadores. Solo un participante integrante del sector de los docentes formadores se ha implicado en el ámbito del currículo integrado (ci).

- Las modalidades presencial y online han sido las más cursadas por los tres sectores de informantes; la de seminario/jornada se ha mostrado como la menos escogida.

De estos resultados, resaltamos la amplia y variada formación continua recibida por el sector de los docentes formadores, cuyas razones justificativas se refieren, por una parte, a los crecientes retos y demandas planteadas para ellos desde los diferentes niveles estructurales y organizativos. Y por otra parte, a la búsqueda de la calidad que han de emprender en el mundo competitivo del empleo, que han cifrado en los distintos ámbitos de formación continua que les son planteados. De lo cual se infiere que, efectivamente, existe en ellos una toma de conciencia del compromiso y responsabilidades profesionales que han de asumir en el ejercicio de su profesión.

Distinto se nos presenta el caso del sector de los docentes educadores; no se involucran homogéneamente en los ámbitos de formación continua, no dando cabida ni al ámbito de los programas europeos (pe) ni al del PE (pel). Situación que podría deberse a la relevancia que -desde las necesidades socioeducativas- se concede a determinados ámbitos de formación, determinándoles a elegirlos con carácter primordial y urgente, entendiendo que su participación les va a permitir ajustar armónicamente la enseñanza. El consiguiente relego de otros ámbitos formativos no hace sino aclamar la necesidad de prestarles la atención que merecen por su equitativa importancia con los demás.

El hecho de que el ámbito de actualización e innovación metodológica/didáctica (imd) resulte ser muy representativo para los tres sectores de informantes, quizá revele la sólida conciencia extendida de instaurarse en un marco permanente de reciclaje profesional y actualización de métodos docentes, en donde puedan tomar contacto y familiarizarse con nuevos modelos o perspectivas emergentes. Sin embargo, si atendemos al componente de innovación que nos plantea el ámbito de formación continua anterior, unido a sus conexiones con el ámbito de la metodología AICLE/CLIL/EMILE ( $\mathrm{ma}$ ) y el del currículo integrado (ci), observamos que tal componente no está cubierto en todas sus vertientes. Exaltamos la necesidad que deben asumir los docentes de LE de contemplar los dos ámbitos de formación continua citados -con independencia de su situación y tiempo, y del contexto en el que desempeñen su trabajo-, como opciones formativas que pueden incidir significativamente en su desarrollo profesional.

Igualmente, se puede observar que tanto la docente doctora como los docentes de mayor edad de los tres sectores de informantes son quienes más tiempo de formación han recibido, predominantemente en modalidad presencial y online. Éstos realmente ejercen la importante función de gestionar los distintos ámbitos de formación continua; función que no es evidente que revierta adecuadamente en la toma de decisiones que realice cada docente de LE para remediar sus necesidades y operativizar sus carencias formativas, a través del itinerario con los ámbitos de formación ofertados. 
Cabe inferir que ha de ser el docente de LE -quien sensibilizado ante su perfil profesional y las condiciones de su situación particular- el que sopese adecuadamente los ámbitos que solventen sus necesidades formativas, al igual que sepa distribuir y compaginar su participación en ellos, según modalidad y tiempo.

\subsection{El componente competencial: competencias profesionales docentes}

Ante los resultados, detectamos que los docentes formadores y los futuros docentes otorgan un mayor valor a aquellas competencias a las que se espera que un docente educador atribuya mayor importancia: la competencia didáctica $(c d)$ o la de gestión de la convivencia (cgc). Se infiere la existencia de discrepancias internas y externas en los docentes educadores, ya que han podido verse abocados a una responsabilidad directa, conforme a la cual tienden a prescindir de aquellas competencias más vinculadas al propio ámbito formativo.

Es preciso tener también en cuenta la débil valoración que los tres sectores efectúan de la competencia digital (ctic) y de la social relacional (csr), cuando actualmente, más se apuesta por ellas en cualquier sector de trabajo. Contradicción que parece derivarse del insuficiente provecho que el profesorado extrae de dichas competencias; causado, a su vez, por una escasa transferibilidad de la formación recibida al aula. Esto se desprende de verificar que la competencia digital es la que más mejora tras la formación continua recibida. Hecho que no sucede, sin embargo, con la competencia social-relacional.

Ante estas constataciones, cabe destacar además las dispares valoraciones que los docentes de mayor edad otorgan a las competencias profesionales, a lo cual puede subyacer un sentido profesional crítico o, contrariamente, una escasa conciencia de las ventajas que supone valorar positiva y equitativamente todo el referencial de competencias profesionales establecidas.

La información generada sobre las acciones pasadas y/o actuales (apa) y futuras (af) emprendidas para la mejora de las competencias profesionales, nos permite hacer referencia a los siguientes aspectos:

a) Los docentes educadores perciben la necesidad de mejorar su competencia comunicativa y lingüística ( $c c l)$, por lo cual, se inscriben en los ámbitos de formación continua correspondientes y emprenden acciones formativas para satisfacer dicha necesidad. De esta consideración se desprende una carencia principalmente lingüística, cuyo origen quizá proviene de la larga tradición monolingüe que -en las pasadas décadas- ha marcado nuestro contexto organizativo curricular. E incluso, de forma específica, de la formación inicial, en la que si bien se les instruye en aspectos curriculares y didácticos -en todo caso generales-, se relega a un segundo plano el desarrollo de competencias relacionadas con las LE y su enseñanza. Esta idea se respalda, a título ilustrativo, ante la presencia de tres asignaturas dedicadas al conocimiento de aspectos lingüísticos de LE y solo de otras tres específicas de didáctica y metodología de LE -frente al resto de las 32 asignaturas que completan el Título ofertado de Grado en Maestro de Educación Primaria de la Universidad de Valladolid. Hecho pendiente de reconsideración por los agentes responsables, para determinar y regular las disciplinas del plan de estudio y ajustar su respectiva carga crediticia. 
Y sobre las acciones futuras que piensa emprender este sector para mejorar sus competencias, sobresale la continuación en los distintos ámbitos de formación continua y viajar al extranjero. Datos que apoyan las consideraciones anteriores relativas a los aspectos lingüísticos, produciéndose, además, una tácita inadvertencia a tratar de mejorar el resto de competencias profesionales docentes.

b) Los docentes formadores manifiestan su voluntad por cultivar conocimientos para la mejora de la competencia científica cognitiva ( $c c c)$, en la perspectiva de la búsqueda de la calidad docente y la presunta finalidad de aproximarse a la excelencia profesional. Aspecto que se corrobora con las acciones futuras expresadas por este sector de informantes, las cuales presumen mantenerse en la misma línea.

c) Los futuros docentes han situado los propios estudios de postgrado entre las acciones actuales en curso de realización, para mejorar sus competencias profesionales, poniendo de relieve la importancia de aprender de la propia experiencia y de continuar formándose.

Se infiere que es de rigor atender a aquellas competencias que han sido objeto de mejora con menor trascendencia -entre los tres sectores informantes, especialmente en los docentes educadores-; es el caso de las competencias: científica cognitiva (ccc), en gestión de la convivencia (cge), la intra e interpersonal (cii), en trabajo en equipo (cte), la organizativa y de gestión de la calidad (cog), y la social-relacional (csr). Coincidentemente, -a excepción de las dos primeras-, se trata de cuatro de las cinco competencias que se han tomado como aquellas que inciden en la autoeficacia percibida del docente de LE. Lo cual nos puede ya indicar bajas percepciones de autoeficacia, cuya solución puede encontrarse en los ámbitos de formación continua, donde la primacía de los contenidos que tratan revierte en la mejora de ciertas competencias que no se vinculan con las de la autoeficacia percibida del docente de LE.

\subsection{El componente de evaluación}

Por un lado, se nos muestra la tendencia de los sectores de informantes a servirse de programas e instrumentos para su propia evaluación docente (pi), así como a obtener certificados de acreditación mediante la realización de pruebas oficiales ( $p o$ ).

Por otro lado, las informaciones generadas por cada sector de informantes sobre la frecuencia y el modo en que utilizan estos programas e instrumentos, dan cuenta de que son muy pocos los docentes educadores y futuros docentes que se sirven de ellos, y de que las aportaciones de sus alumnos son sus principales fuentes de feedback. Por el contrario, la mayor parte de los docentes formadores se sirve de encuestas, si bien con diferencias en la frecuencia de uso. Estos resultados nos permiten inferir la falta de auténticas autopercepciones -en el sector de los docentes educadores-, sobre las condiciones de su desarrollo profesional, ya que la evaluación y autoevaluación son dos grandes pilares para la transformación de las prácticas de enseñanza (Perrenoud, 2008) que dan cuenta de los atributos que influyen en la mejora de sus competencias profesionales.

Por lo que se refiere a la actualización de certificados de acreditación en LE a través de pruebas oficiales, los resultados obtenidos de los tres sectores informantes se presentan 
con mayor homogeneidad, pudiéndose afirmar que la mitad de los informantes pasa pruebas oficiales para esa actualización, lo cual tiene mayor sentido en el caso de los docentes educadores, quienes ya habían manifestado la necesidad de mejorar su competencia comunicativa y lingüística. Sentido que también encontramos en el caso de los docentes formadores y los futuros docentes, ya que ciertas características de éstos - caso de la edad-, inducen a la lógica de utilizar pruebas oficiales para obtener y actualizar sus certificados lingüísticos de acreditación.

\subsection{El componente de eficacia}

El registro y análisis de los datos recogidos a través de una escala Likert, que se ajustan a la Categoría de análisis 3. La Autoeficacia percibida, nos permite presentar los resultados optando por el sistema de frecuencia de aparición de los ítems. Recogemos en la Tabla 1 los resultados, como aspecto previsor de las reflexiones contempladas en el apartado de las Conclusiones:

Tabla 1. Identificación del estado de las competencias profesionales de los sectores informantes conforme a su sentimiento de eficacia percibida

\begin{tabular}{|c|c|c|c|c|c|c|}
\hline $\begin{array}{c}\text { Unidades de } \\
\text { análisis }\end{array}$ & $\mathbf{C P}$ & $\boldsymbol{C} / \boldsymbol{I I}$ & $\boldsymbol{C} / \boldsymbol{O G}$ & $\boldsymbol{C} / \boldsymbol{T E}$ & $\boldsymbol{C} / \boldsymbol{M}$ & $\boldsymbol{C} / \boldsymbol{S R}$ \\
\hline Docentes educadores & Muchas veces & Siempre & Muchas veces & Muchas veces & Muchas veces \\
\hline Docentes formadores & Siempre & Siempre & Muchas veces & Siempre & Muchas veces \\
\hline Estudiantes del Máster & Siempre & Muchas veces & Algunas veces & Muchas veces & Algunas veces \\
\hline
\end{tabular}

En los docentes educadores, los resultados nos muestran unos índices muy altos en la autopercepción del estado de los componentes que engloban sus competencias profesionales -tomadas como subcategorías de la categoría principal de análisis La autoeficacia percibida-, (a excepción de la parte inter de la competencia intra e interpersonal (C/II). Parte que comprende conocimientos, destrezas y actitudes que giran en torno a la orientación, las propias limitaciones, la tutorización/mentorización y el feedback por parte de colegas de profesión (Modelo JCyL, 2011a; EPG, EAQUALS, 2013). Estos aspectos son, por lo tanto, objeto de mejora de la competencia interpersonal para este sector.

Las altas atribuciones que ha efectuado el sector de los docentes formadores nos continúan advirtiendo de un sector sólidamente formado, con alta consciencia y expectativas para continuar desarrollándose profesionalmente.

$\mathrm{Y}$ el sector de los futuros docentes refleja igualmente altos índices sobre los componentes de las competencias profesionales. La competencia en trabajo en equipo $(C / T E)$ y la competencia social-relacional $(C / S R)$ no alcanzan el máximo valor, hecho que no coincide, a priori, con lo que se espera del sector de los futuros docentes, puesto que desde la formación inicial son competencias que, en virtud de la esencia de Bolonia, están siendo objeto de desarrollo en las enseñanzas universitarias. Sin embargo, puede bien entenderse si tenemos en cuenta primero los aspectos señalados en el análisis descriptivo, tales como los estudios previos y la ocupación profesional, y después, los enunciados que recogen los componentes de estas dos competencias. 
Inferimos la existencia de una carencia en los docentes de LE, tanto de conocimiento como de visión crítica, para cuyo remedio se consideraría idónea la asignación de la figura de un asesor externo de calidad. Asesor tal como un mentor o un orientador (por las propias características de la competencia interpersonal), procedente de la Universidad o de los Departamentos de Orientación de los respectivos centros, cuya función se sitúe en el acompañamiento al docente de LE en su proceso de toma de decisiones y la guía en su proceso formativo.

\section{Conclusiones}

De manera reflexiva, las conclusiones constituyen aquí un conjunto de aportaciones, afirmaciones, respuestas o tentativas de respuestas que se pretenden con efectos sobre el desarrollo de las competencias profesionales de los docentes de LE.

Las referencias abordadas nos confirman que, para la mejora de la calidad educativa de los individuos, los docentes de LE -en virtud del principio de subsidiariedad que preside la Educación y la Formación en los estados miembros de la Unión Europea-, son los responsables últimos. Lo cual conlleva, por una parte, la toma de conciencia de la importancia de su propio perfil profesional docente, en virtud a su vez de las competencias profesionales que deben poseer. Y, por otra parte, las acciones pertinentes como responsables de su propia profesionalización y desarrollo profesional, en virtud de los principios que sustentan el $E P G$, conforme a las determinaciones europeas sobre evaluación y acreditación de competencias profesionales a lo largo de la vida.

Ante los resultados obtenidos y su interpretación, se revelan las siguientes conclusiones que, sin ánimo de generalizar, entendemos pertinentes para la especificidad de los sectores de docentes contemplados:

- La atención al sentimiento de eficacia personal -concretado en la autoeficacia percibida- se muestra como un "lugar" de indagación muy productivo, ante las evidencias generadas sobre los rasgos y características de la configuración del desarrollo profesional de los docentes de LE.

- La complementariedad de la sensibilización ante su propio perfil profesional y de las autopercepciones sobre las condiciones de configuración de su desarrollo profesional se convierte en una estrategia de profesionalización de estos docentes de LE, en diversas direcciones:

a) Una equitativa atribución de importancia a todos los ámbitos de formación continua satisfará las intenciones de desarrollar por igual el referencial de competencias profesionales planteadas para el docente de LE.

b) La integración de los ámbitos de formación continua relativos a la metodología AICLE/CLIL/EMILE y al currículo integrado, puede ampliar significativamente el campo de las acciones que pueden ser emprendidas por el docente de LE, para contribuir a la mejora de la calidad educativa.

c) Una buena selección de los ámbitos de formación continua planteados para el docente de LE y una buena gestión de los mismos a través de la adecuada 
elección de modalidad y tiempo, puede operativizar la capacidad de su recorrido formativo e influirle en el rendimiento disciplinar para su excelencia profesional.

d) Una valoración equitativa de las competencias profesionales propias del docente de LE implicará la distribución equitativa en la elección, para cursar los ámbitos de formación continua planteados.

e) La contribución a la mejora de las competencias profesionales del docente de LE sería efectiva si, desde la formación inicial, se abarcase todo el espectro disciplinar focalizado en desarrollar las competencias profesionales de los futuros docentes, enfatizando en aquellos ámbitos que sienten sólidamente las bases y permitan remediar las carencias competenciales actuales percibidas, tanto en comunicación lingüística como en las de los otros componentes analizados.

f) La facilitación de oportunidades y la disposición de medios necesarios para la formación continua eficaz de los docentes educadores en LE reducirán las interferencias que encuentran en su desarrollo profesional.

g) La utilización de programas e instrumentos de evaluación de forma asidua, por una parte, profesionalizaría al docente de LE, al permitirle "visualizar" el estado de sus competencias e identificar sus necesidades de formación; $y$, por otra parte, regularizaría positivamente el nivel de autopercepciones que posee sobre las condiciones de su desarrollo profesional.

- La autoeficacia percibida del docente de LE regula su toma de decisiones para involucrarse en los distintos ámbitos de formación continua y en aquellas acciones destinadas a mejorar sus competencias profesionales, desde el punto de vista de su sistema propio de factores personales, externos e internos.

- Los planes formativos y la toma de decisiones del docente de LE sobre su desarrollo profesional requieren de acompañamiento - de una asesoría de alta calidad-, en función de las diversas situaciones de enseñanza, del tiempo de docencia y de las informaciones generadas en torno a la autoeficacia percibida.

- El productor como "mecanismo generador" y el producto como "objeto resultante" de una oferta y diseño de planes de formación, así como de las acciones de un profesional -un "asesor externo de calidad"-, sobre el itinerario de profesionalización y desarrollo profesional del docente de LE se encuentran en la autoeficacia percibida.

\section{REFERENCIAS BIBLIOGRÁfiCAS}

Bandura, A. (2003). Auto-efficacité. Le sentiment d'efficacité personnelle. Bruxelles: De Boeck. Bandura, A. (2006). "Guide for constructing self-efficacy scales", in F. Pajares y T. Urdan (eds.), Self-efficacy Beliefs of Adolescents. Greenwich, Connecticut: Information Age Publishing, 307-337.

Bardin, L. (2002). Análisis de contenido. Madrid: Akal.

Beckers, J. (2007). Compétences et identités professionnelles. L'enseignement et autres métiers de l'interaction humaine. Bruxelles: De Boeck.

European Commission, (2008). Eurotrainer - Making lifelong learning possible: A study of the situation and qualifications of trainers in Europe. Brussels: European Commission. Di- 
rectorate General for Education and Culture. Available from http://ec.europa.eu/education/ more-information/doc/eurotrainer1_en.pdf accessed 15 April, 2014.

Donnay, J. y Charlier, E. (2008). Apprendre par l'analyse des pratiques: Initiation au compagnonnage réflexif. Namur: Presses Universitaires de Namur.

EAQUALS (2013). Parrilla del perfil del profesor de idiomas [versión 0.44]. Disponible en http:// www.epg-project.eu/wp-content/uploads/The-EPG-PDF-publication_EN.pdf consultado el 18 de enero de 2014.

Flick, U. (2004). Introducción a la investigación cualitativa. Madrid: La Morata.

Gurdián-Fernández, A. (2007). El Paradigma Cualitativo en la Investigación Socio-Educativa. Costa Rica: IDER, CECC, AECI.

Huberman, A. y Miles, M. B. (1994). Qualitative data analysis. Thousand Oaks: Sage.

INCUAL (2007). Sistema Nacional de Cualificaciones y Formación Profesional. Madrid: Ministerio de Educación, Política Social y Deporte/ Instituto Nacional de las Cualificaciones.

JCyL (2011a). Modelo de competencias profesionales del profesorado. Madrid: Centro Superior de Formación del profesorado. Dirección General de Calidad, Innovación y Formación del Profesorado.

JCyL (2011b). Plan de Formación del Profesorado en Lenguas Extranjeras. Madrid: Centro Superior de Formación del Profesorado en Idiomas. Dirección General de Calidad, Innovación y Formación del Profesorado.

Kelly, M. et al. (2004). European Profile for Language Teacher Education. A Frame of Reference. Southampton: University of Southampton.

Krippendorff, K. (1990). Metodología del análisis de contenido. Teoría y práctica. Barcelona: Paidós Ibérica, S.A.

Marcelo, C. (1987). El pensamiento del profesor. Barcelona: CEAC.

Paquay, L. et al. (dir.). (2010). Former des enseignants professionnels. Quelles stratégies? Quelles compétences. Bruxelles: De Boeck.

Perrenoud, P. (2008). La evaluación de los alumnos. De la producción de la excelencia a la regulación de los aprendizajes. Entre dos lógicas. Buenos Aires: Colihue.

Pilhion, R. (2008). "Vers une politique européenne de l'enseignement des langues", in Revue Internationale d'Éducation de Sèvres, 47: 27-35.

Stake, R. (2010). Qualitative Research: Studying how things work. Nueva York: The Guilford Press.

Tardif, M. (2004). Los saberes del docente y su desarrollo profesional. Madrid: Narcea.

Tejada, J. (2013): "Profesionalización docente en la universidad: implicaciones desde la formación. La informalización de la educación" [monográfico en línea]. Revista de Universidad y Sociedad del Conocimiento (RUSC). 10, 1: 170-184. Disponible en: http://rusc.uoc.edu/ojs/ index.php/rusc/article/view/v10n1-tejada/v10n1-tejada-es consultado el 3 de marzo de 2014. 
